\title{
Drivers learn city-scale dynamic equilibrium
}

\author{
Ruda Zhang and Roger Ghanem \\ Department of Civil and Environmental Engineering, \\ University of Southern California, Los Angeles, CA, USA.
}

(Dated: August 24, 2020)

\begin{abstract}
Understanding collective human behavior and dynamics at urban-scale has drawn broad interest in physics, engineering, and social sciences. Social physics often adopts a statistical perspective and treats individuals as interactive elementary units, while the economics perspective sees individuals as strategic decision makers. Here we provide a microscopic mechanism of city-scale dynamics, interpret the collective outcome in a thermodynamic framework, and verify its various implications empirically. We capture the decisions of taxi drivers in a game-theoretic model, prove the existence, uniqueness, and global asymptotic stability of Nash equilibrium. We offer a macroscopic view of this equilibrium with laws of thermodynamics. With 870 million trips of over 50k drivers in New York City, we verify this equilibrium in space and time, estimate an empirical constitutive relation, and examine the learning process at individual and collective levels. Connecting two perspectives, our work shows a promising approach to understand collective behavior of subpopulations.
\end{abstract}

Keywords: urban-scale dynamics, collective behavior of individuals, human behavior change, social physics

\section{INTRODUCTION}

Quantitative understanding of collective human behavior and its dynamics has been the focus of a long-standing, interdisciplinary collection of research. Such efforts span across physics [1-15], engineering [16-20], economics [2127], and planning [28-30]. Cities, as agglomerations of human beings, are the natural setting for such studies. Thanks to ubiquitous data sensing techniques, a host of urban phenomena, ranging from traffic $[5]$ to building energy use [30] and transaction patterns [31], can now be analyzed quantitatively. Research in the physical sciences often value simplistic models that fit data well. Social sciences, on the other hand, emphasize individual incentive and realistic theory. Models that connect these perspectives and are backed by empirical data would therefore be a valuable contribution to this literature.

Social physics [1] or social dynamics [7, 9, 10] are approaches that use physical models to describe collective human behavior. A useful framework for this is statistical physics, where individuals are seen as elementary units and collective phenomena emerge from their interactions [10]. Random walk models are used to study human mobility at individual level [4]. Non-equilibrium phase transition is observed in the transition of urban traffic from free flow to congestion [6]. Scaling laws of socioeconomic and infrastructure indicators on population have been discovered and generative mechanisms proposed [2, 11]. Based on non-equilibrium statistical physics, sociodynamics models the evolution of city configuration with a master equation [7], and finds application in studying the impact of transportation system on regional development [8]. Entropy models have been used to study spatial structure and interaction [29]. Other tools in statistical

\footnotetext{
* rudazhan@usc.edu; also at the Statistical and Applied Mathematical Sciences Institute, Durham, NC, USA.
}

physics such as spin-like models also find use in the science of cities [14]. Other theories in physics have been adopted as well. Social force and social field models quantify the most probable behavioural change of individuals [9]. Force fields resulting from infrastructure and socioeconomic layouts affect urban morphology [12]. Gravity and radiation have inspired models for human mobility at population level [13]. In particular, a potential field can be well-defined from commute flows in a city [15].

Game theory and economics, on the other hand, consider collective phenomena as results of strategic decision making of individuals, and it is therefore crucial to capture individual incentives realistically. Discussion on traffic equilibrium predates modern game theory [21, 22], and has been formalized since [16, 23, 24]. Spatial equilibrium generalizes this idea to explain many problems in urban economics [25]. Game theory has also been used to study social network formation [3]. Equilibrium outcomes are often different from social optimal and there are many efforts to quantify such inefficiency [18, 19] and find ways to minimize it [5].

These perspectives from physical and social sciences rarely come together, partly because it is difficult to account for the different incentive structures within a population and provide a simplistic, universal understanding. Consider urban transportation. People move for various reasons and often do not minimize their travel time as assumed in traffic equilibrium. But a model that accounts for heterogeneous incentives often evades an equilibrium solution. Here we bridge the two perspectives by studying the behavior and dynamics of a subpopulation - taxi drivers. As ride service providers and independent contractors, these drivers move around a city to maximize income. Using GPS data, recent work has shown that their revenue are affected by their strategies in search, delivery, and service-region preference [20]. Gender earnings gap among drivers of a ride-hailing company is explained by their driving speed, experience, and service location and time [26]. Moreover, drivers with higher incomes ben- 
efit significantly from their ability to learn from local and global demand information [27].

In this paper, we regard the regularity in urban transportation as the equilibrium outcome of individual decision making in response to transportation demand and services [32]. In particular, we provide a microscopic, game-theoretic model for taxi transportation and interpret its equilibrium in a macroscopic framework of thermodynamics. Given exogenous traffic speeds and passenger demand, the income maximization of drivers leads to an economic equilibrium. We formalize drivers' decision making as a non-cooperative game, prove the existence and uniqueness of its Nash equilibrium, and show its stability under simple learning dynamics such as adaptive learning and social learning. We also provide an interpretation of this equilibrium as a thermodynamic equilibrium, and describe the laws of thermodynamics, constitutive relation, and fundamental thermodynamic relation. With five years of New York City (NYC) taxi trip records, we validate the equilibrium in space and over time, and estimate an empirical constitutive relation. We also examine the learning process of individual drivers, as well as their adjustment as a group to a new system. We discuss the economic efficiency of taxi transportation, as well as an alternative formulation of strategy. This paper therefore offers a microscopic mechanism of city-scale dynamics, and empirically verify its various implications. Connecting social and physical science perspectives, our work shows a promising approach to understand collective human behavior and dynamics.

\section{RESULTS}

\section{A. Game model}

We assume that each driver chooses their driving strategy to maximize income, which we show to be strategically equivalent to revenye maximization. Taxi activity varies in space (see fig. 1a-b), and we define the strategy of a driver in service to be how they allocate their service time across the city. Let $s_{i x}$ be the proportion of service time driver $i$ allocates on street segment $x$ and $E$ be the set of street segments in the road network, then the driver's strategy is $\mathbf{s}_{i}=\left(s_{i x}\right)_{x \in E}$, whose components sum to 1. Figure 11c illustrates driver strategy. We can formalize a driver's decision as an optimization problem:

$$
\begin{array}{ll}
\operatorname{maximize} & \pi_{i}\left(\mathbf{s}_{i} ; \mathbf{s}_{-i}, \mathcal{E}\right) \\
\text { subject to } & \mathbf{s}_{i} \geq 0 \\
& \mathbf{s}_{i} \cdot \mathbf{1}=1
\end{array}
$$

Here, with hour as the unit of time, $\pi_{i}$ is the expected hourly revenue of the driver, $\mathbf{s}_{-i}=\sum_{j \neq i} \mathbf{s}_{j}$ is the aggregate strategy of other drivers, and $\mathcal{E}$ is environment condition which includes traffic speed and passenger demand. For details of driver decision making, see Supplementary Note A 1 .
Competition among drivers could lead to specific choices of strategies, called equilibrium. If we see every street segment as a distinct market and every driver in service as a multi-market firm, we can abstract eq. (1) as a game of multi-market competition among firms of equal capacity. This game has a unique Nash equilibrium (NE), where all drivers use the same strategy and marginal driver revenue are uniform across all searched segments. Moreover, the equilibrium is globally asymptotically stable under adaptive learning [33] and/or imitative learning [1, 34, 35]. Denote this equilibrium as $S^{*}=\left(\mathbf{s}_{i}^{*}\right)_{i \in N}$, where $N$ is the set of drivers in service. Because all drivers use the same strategy, let $\mathbf{s}=\sum_{i \in N} \mathbf{s}_{i}$, we can write $S^{*}=n^{-1} \mathbf{s}^{*} \mathbf{1}_{n}^{\mathrm{T}}$, where $n=|N|$ is the number of drivers in service. The equilibrium can be determined such that $\mathbf{s}^{*}$ is the unique point that maximizes a potential function:

$$
\Phi(\mathbf{s})=\sum_{x \in E} \int_{0}^{s_{x}} \phi_{x}(t) \mathrm{d} t
$$

where $\phi_{x}\left(s_{x}^{*}\right)=\left(\partial \pi_{i} / \partial s_{i x}\right)\left(S^{*}\right)$ is marginal driver revenue on a segment at equilibrium, see fig. 11. For details of the game model, see Supplementary Note A 2 .

\section{B. Thermodynamic interpretation}

We can interpret the Nash equilibrium of drivers as a thermodynamic equilibrium. This establishes a macroscopic equilibrium where aggregate behavior is perceived as a transport phenomenon built up from individual choices. This macroscopic view ignores the decision making and competition of drivers, but helps understand the outcome of a social system from the perspective of a physical system.

We regard drivers as interchangeable particles with identical behavior at equilibrium. Regard total service time $s$, which equals the number of drivers in service, as total energy of the taxi transportation system. Regard potential function $\Phi$ of the game as entropy of the system. And regard the reciprocal of equilibrium marginal driver revenue, $\psi=1 / \phi$, as temperature. Then $s, \psi$, and $\Phi$ are all state variables of the system at equilibrium given environment condition $\mathcal{E}$.

Being a state variable and intensive property, temperature $\psi$ is the driving force of the transport of service time $s$ over the street segments. As we mentioned earlier, the learning process of the game always increases the potential function $\Phi(\mathbf{s})$, which is maximized at equilibrium. When two systems at equilibrium are put into contact with an interface permeable to the transfer of service time, $s$ will flow from the system with higher $\psi$ to the one with lower $\psi$. At equilibrium, $\psi$ is uniform across all searched segments. In summary, we can make the following statements of thermodynamics. Zeroth law: two taxi systems in contact have the same equilibrium 

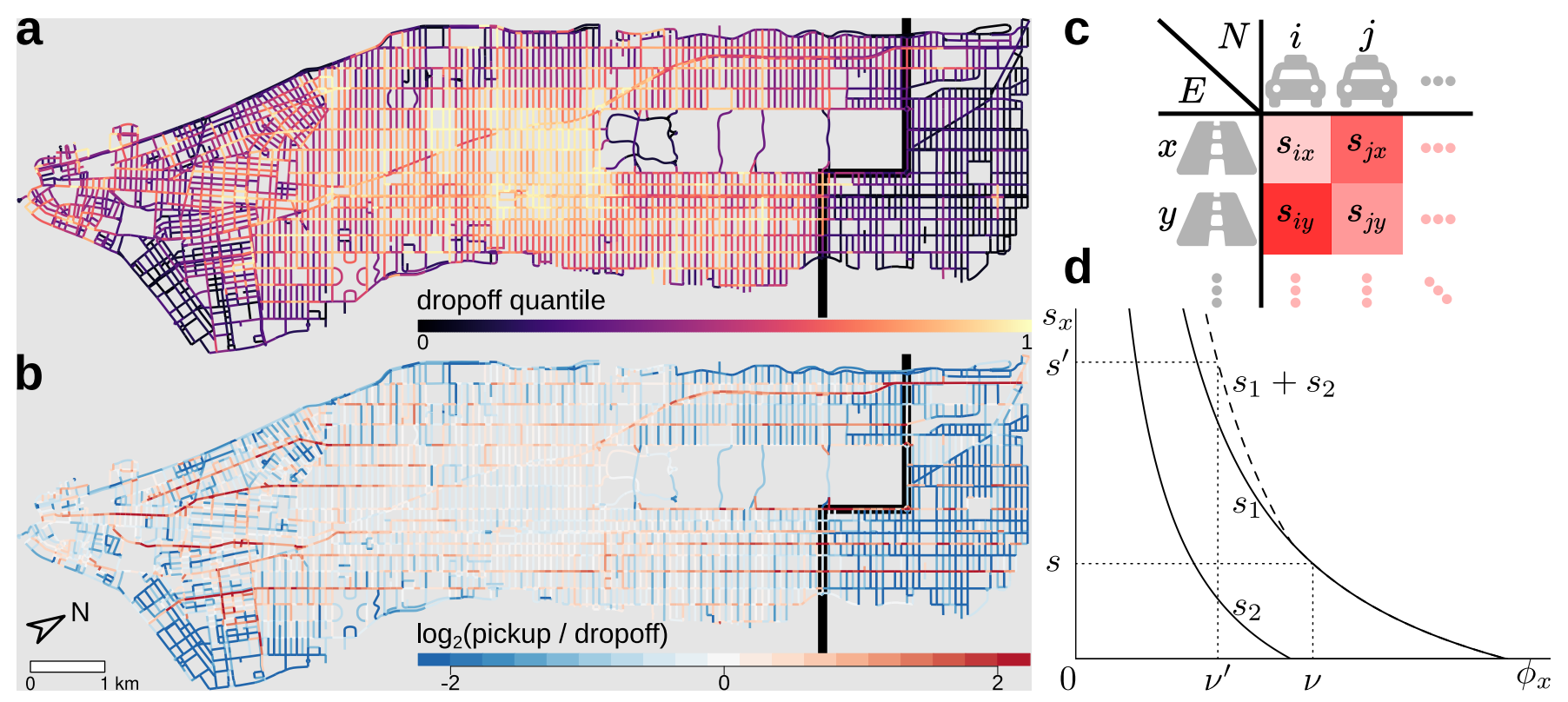

FIG. 1. Driver strategy. a-b, Manhattan street network used in this paper, showing characteristics of taxi activity: a, quantile of drop-off per segment length; b, log2 pickup-dropoff ratio. Black bold line marks the north border of core Manhattan. c, drivers allocate their service time across the segments, which can differ. d, model of equilibrium. With $s$ drivers, marginal driver revenue $\nu$ on a segment at equilibrium is determined by $\sum_{x \in E} s_{x}(\nu)=s$. Equilibrium allocation on each segment can then be determined by $s_{x}^{*}=s_{x}(\nu)$.

marginal driver revenue.

$$
\psi_{1}=\psi_{2}
$$

First law: taxi transportation is the transfer process of total service time $s$, which is a conserved quantity.

$$
\mathrm{d} s=\sum_{x \in E} \mathrm{~d} s_{x}
$$

Second law: under fixed demand and traffic state, a closed taxi system maximizes its potential function.

$$
\mathrm{d} \Phi \geq \frac{\delta s}{\psi}
$$

Zeroth law defines equivalent classes of equilibrium, which are strictly totally ordered by state variable $\psi$. The manifold of equilibrium is thus one-dimensional, parameterized by $\psi$, and any other state variable must depend on it. This means that state space $(\Phi, s, \psi) \mid \mathcal{E}$ has only one degree of freedom, and this dependency is the constitutive relation of the system given environment condition $\mathcal{E}$, which can be written explicitly as

$$
(\Phi, \psi)(s) \mid \mathcal{E}
$$

Rearranging the exact differential of $s(\Phi) \mid \mathcal{E}$ gives the fundamental thermodynamic relation of the equilibrium:

$$
\mathrm{d} s=\psi \mathrm{d} \Phi
$$

We test various implications of this theory of thermodynamics in our empirical results.

\section{Verification of spatial equilibrium}

To verify that drivers actually follow the theoretical equilibrium, we proceed in two parts. First, all drivers use the same strategy. Second, given that drivers use the same strategy, marginal driver revenue is uniform across all searched segments.

Although driver strategy - the spatial distribution of service time - is not directly observed, it is proportional to driver pickup probability on each segment. If all drivers use the same strategy, each driver's pickup probability distribution across segments shall be the same as that of the overall distribution. Then each driver's actual pickups shall be a sample of the corresponding categorical random variable. Since there are 6,001 street segments, pickup records of each driver is not enough to test the probability model. We partition the segments into 10 equi-probable groups, so pickup counts in these groups shall be a multinomial random variable with the same probability for each group. Drivers' pickup counts in these groups can be tested by a corrected log likelihood ratio of multinomial distributions [36]. For each driver, the pickup counts are normalized into a probability vector $\mathbf{x}=\left(x_{1}, \ldots, x_{10}\right)$, which is then summarized by the 1-norm $\left\|\mathbf{x}^{\prime}\right\|_{1}$, where $\mathbf{x}^{\prime}=\mathbf{x}-\mathbf{1} / 10$. We consider a strategy to be a large deviation if $\left\|\mathbf{x}^{\prime}\right\|_{1}$ exceeds 0.3 . Note that $\left\|\mathbf{x}^{\prime}\right\|_{1}=0.2$ if the pickups in any one group is arbitrarily allocated to the other groups. Fig. 2a shows the distribution of $\left\|\mathbf{x}^{\prime}\right\|_{1}$. Only $3.66 \%$ of drivers have statistically significant large deviations. Although the threshold for large deviation is arbitrary, the result shows that most 
a

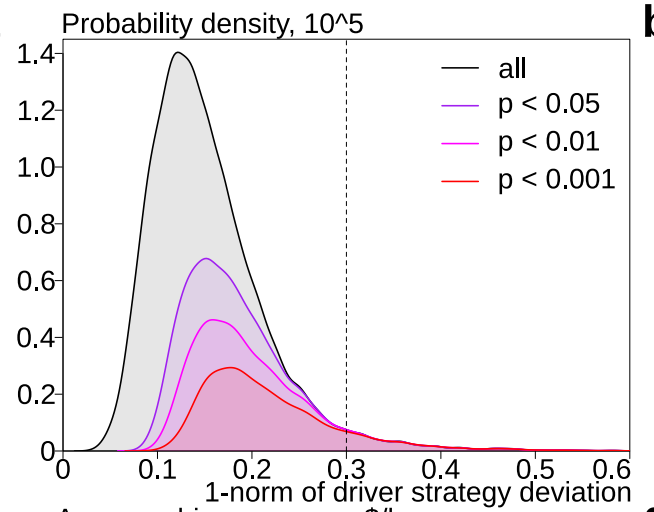

C

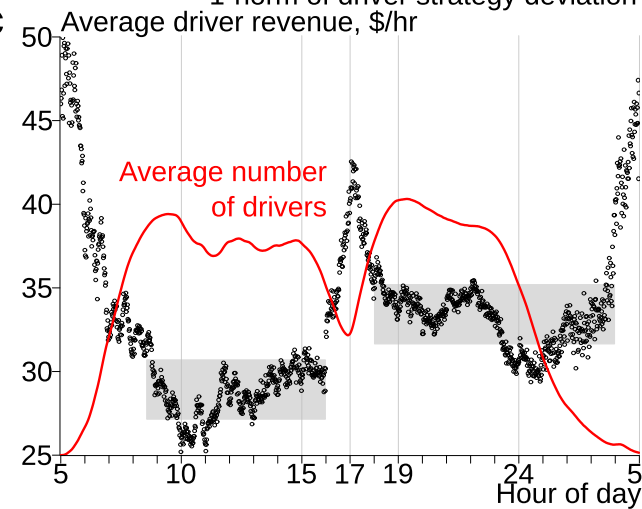

b Estimated search time-revenue ratio, log, shifted

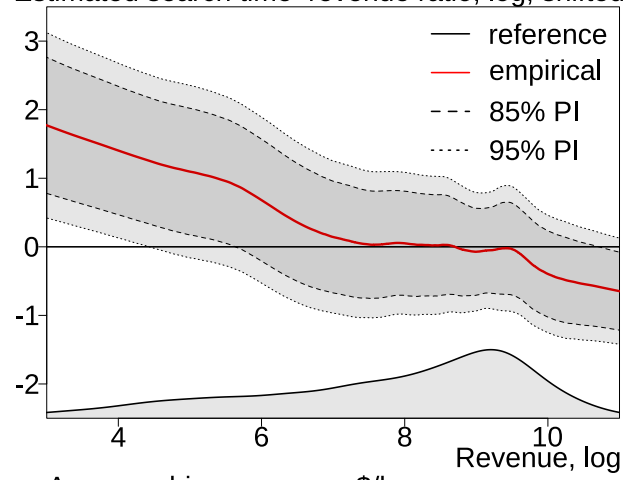

$\mathbf{d}_{50}$ Average driver revenue, $\$ / \mathrm{hr}$

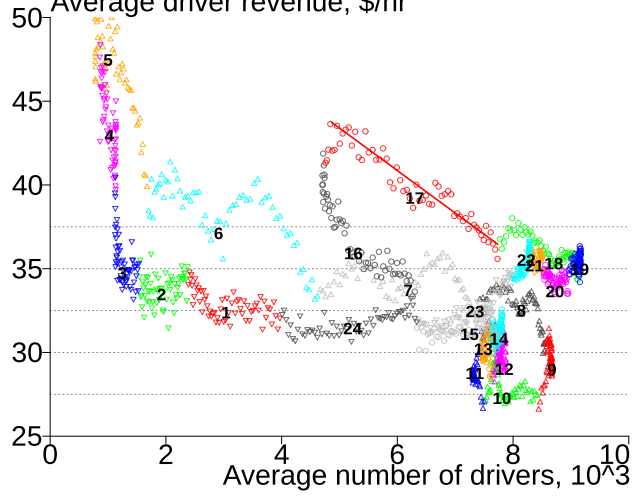

FIG. 2. Verification of dynamic equilibrium. Using trip records in Spring 2011 and Spring 2012. a, probability distribution of 1-norm of driver deviation from average strategy, in Tue-Thu PM peaks, $6 \mathrm{pm}-10 \mathrm{pm}$, grouped by $p$-values. $3.66 \%$ of drivers have statistically significant $(p>0.05)$ large deviations $\left(\left\|\mathbf{x}^{\prime}\right\|_{1}>0.3\right)$. b, log of search time-revenue ratio on street segments, Mon-Fri $6 \mathrm{pm}-7 \mathrm{pm}$, shifted to a reference value. Local regression (red) and prediction intervals (shade). Distribution of log revenue on segments (margin). c-d, average number of drivers and driver revenue, Wed 5am-Thu 5am: c, time series, rectangles mark AM shift (8:30am-4pm) and PM shift (6pm-4am); d, trajectory, colored by the hour, red line shows a linear regression for 5pm.

drivers use similar strategies. Therefore we can regard drivers to be particles with identical behavior.

Now we verify that all segments have the same marginal driver revenue, or equivalently, the same temperature. We note that when $n \gg 1, \phi_{x} \approx \pi_{x} / s_{x}$, where $\pi_{x}$ is the revenue originated on a segment and $s_{x}$ is the total service time attributable to the segment. Because at any moment the number of drivers in service in Manhattan is in the thousands, this approximation is suitable. So it suffices to show that $\pi_{x}$ is proportional to $s_{x}$, which is the sum of search time $t_{s x}$ and trip time $t_{p x}$ per unit time. Because the majority of trips are metered, which is calculated from trip distance and time in slow traffic, driver revenue from each trip is highly correlated to trip duration regardless of driver strategy, especially when traffic speed is hold stationary. To avoid the influence of this fact, consider trip time as a linear function of trip revenue, then $\pi_{x} \propto s_{x}$ is equivalent to $\pi_{x} \propto t_{s x}$, and we try to show the latter. Because search routes are not recorded in the trip records, we take trip records between $6 \mathrm{pm}$ and $7 \mathrm{pm}$ on weekdays in spring, and estimate search routes between trips by shortest distance routing. We consider this approach acceptable because during the selected hours, traffic is roughly at a uniform congested speed while average search time is the shortest, so route deviation from the shortest path is unlikely. Figure 2b shows $\log \left(\tilde{t}_{s x} / \pi_{x}\right)$ versus $\log \left(\pi_{x}\right)$, where $\tilde{t}_{s x}$ is the estimated search time. The majority of street segments have similar search-revenue ratios, while segments with low revenue appear to be over-supplied and those with very high revenue under-supplied. For segments with low revenue, marginal driver revenue might not be equilibrated since they contribute little to driver revenue. Our estimation assigns search time equally to each segment on route, which may underestimate the actual search time near the pickup location, and therefore underestimate search time on high revenue segments. Moreover, shortest path routing provides a single route for trips with the same origin and destination, so the estimated search time may be concentrated on a few street segments, which contributes to estimation error.

\section{Dynamic equilibrium}

As environment condition $\mathcal{E}$ varies over times of a day, the equilibrium will also vary. If drivers are free to choose when to work and are indifferent about work- 

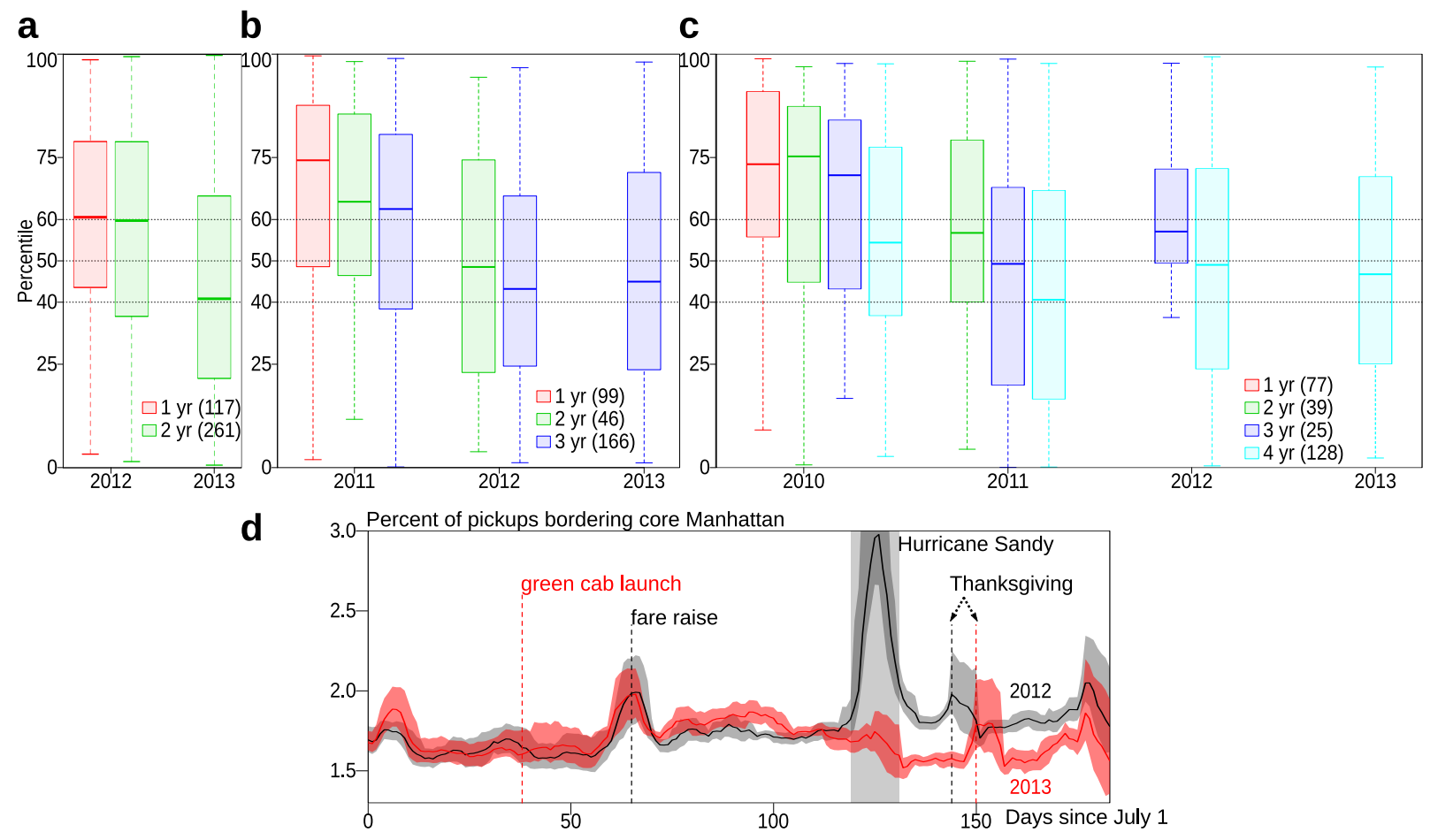

FIG. 3. Learning and adjustment of equilibrium. a-c, percentile of strategy deviation of new drivers joined in a 2012, b 2011, and c 2010, grouped by years of consecutive driving up to 2013, size of each group shown in parentheses. Using trip records in springs, Tue-Thu PM peaks, 6pm-10pm. d, pickup probability in the region bordering core Manhattan, in the second halves of 2012 (black) and 2013 (red), 7-day rolling value with 90\% bootstrap confidence band. Significant events and period marked by dashes and shade.

ing at different times of a day, by zeroth law eq. (3), driver supply $s$ will adjust so that temperature $\psi$ is stationary throughout a day. Equivalently, $\phi$ stays the same throughout a day. Note that marginal driver revenue on a segment and average driver revenue are approximately the same at equilibrium: because $\phi_{x} \approx \pi_{x} / s_{x}$, therefore $\phi \approx \sum_{x} \pi_{x} / \sum_{x} s_{x}=\pi / s$. This means that, given the assumptions, average driver revenue is the same throughout a day. To verify this, we examine the trajectory of average driver revenue and number of drivers throughout a typical weekday, shown in Fig. 2c. Average driver revenues during 8:30am-4pm and $6 \mathrm{pm}-4 \mathrm{am}$ center around $\$ 29 /$ hour and $\$ 33.5 /$ hour respectively, and are constant in the sense that its overall variation is about the same as its short-term variation. The difference between average driver revenue for these two periods can be explained by two factors. First, the total number of taxis is limited and not all is available for the night shift, so not all drivers who would like to work at night can get a taxi. Second, the lease rate for day shifts is less than those of night shifts, so the difference in average driver income between the two periods is less than that of average driver revenue. During $4 \mathrm{pm}-6 \mathrm{pm}$ most double-shifted taxis change drivers, which means supply decisions during this period is not up to the drivers, so the average driver revenue is not constant. During 3am-6am very few drivers are at work, and the high average driver revenue justifies the cost of working when most people prefer to be sleeping. During 6am-8:30am most day shift drivers start working, and although the average driver revenue is not constant, it stabilizes as more drivers become active.

In contrast to the equilibration of average driver revenue over time, by constitutive relation eq. (6), marginal driver revenue on a segment at equilibrium is a decreasing function of the number of drivers given environment condition: $\phi(s) \mid \mathcal{E}$. This constitutive relation is hard to measure without controlled experiment, but can be measured from observational data if the number of drivers is forced to change much faster than the environment does, such as during shift transition. In fig. 2d, the downward trend in $5 \mathrm{pm}-6 \mathrm{pm}$ reflects $\phi(s)$ for that time of day, when people leave work and taxis return for the night shift.

\section{E. Individual learning}

It is natural to ask if drivers learn to use the same strategy that results in a spatially uniform marginal revenue. We use drivers' first appearance in trip records to infer if they are new or experience drivers. The rate of new drivers stabilizes around September 2009, with about 10.23 new drivers each day since. For new drivers joined each spring from 2010 to 2012, we compute the 1-norm of their strategy deviation, $\left\|\mathbf{x}^{\prime}\right\|_{1}$, and compare 
it with the group of experienced driver who worked through 2010-2013. In particular, we group each year's new drivers by their eventual consecutive years of driving up to 2013, and track their percentile of $\left\|\mathbf{x}^{\prime}\right\|_{1}$ against the experienced drivers. Figure 3 a-c provide box plots for the groups. Note that the experienced drivers, if plotted, would always have the median and the first and third quartiles at 50, 25, and 75, respectively. For all groups of new drivers who stayed for at least a year, their strategy deviation decrease significantly in the second year, with the median reducing between 10 to 20 percentile. For new drivers who stayed through 2013 and for at least two years, their strategy deviation stabilize in the later years and are smaller or the same as the experienced drivers. Moreover, new drivers who stay longer always have smaller strategy deviation than their cohorts. We see that new drivers learn the equilibrium strategy within one year of driving.

\section{F. Group adjustment}

Changes in taxi regulation affect the equilibrium, which provide unique opportunities to test the implications of the theory. On 2013-08-08, NYC TLC launched Street Hail Livery, also known as green cabs. The new system is allowed to pick up street-hail passengers outside core Manhattan, defined as south of West 110th Street and East 96th Street, see fig. 1a-b. This change gradually increased the supply of street-hail service outside core Manhattan, and by constitutive relation eq. (6) this should decrease the marginal driver revenue on segments therein. By zeroth law eq. (3), segments within core Manhattan should also have marginal driver revenue decreased to the same level, which implies more supply of yellow cabs in core Manhattan where they have exclusive rights to service. By first law eq. (4), the proportion of service time yellow cab drivers spent outside core Manhattan should decrease. Figure 3d compares the time-series of percentage of pickups in the region bordering core Manhattan in 2012 and 2013. This percentage slightly reduced after the 2012 fare raise, greatly increased during Hurricane Sandy, and moderately increased during Thanksgiving and Christmas. Excluding irregularities due to Hurricane Sandy and the holidays, the percentage is stable in the last two months of both years, with a robust decline in 2013.

\section{DISCUSSION}

The equilibrium is not socially optimal in general. A socially optimal outcome would maximize total revenue, whereas the equilibrium maximizes the potential function. In fact, if total revenue is maximized, marginal segment revenue $\partial \pi_{x} / \partial s_{x}$ should be the same for all searched segments. At Nash equilibrium, a weighted average of marginal and average segment revenue is made uniform instead, with more weight on the latter as the number of drivers in service increases. This difference implies an inefficiency of the equilibrium, except for special choices of $\pi_{x}\left(s_{x}\right)$. For details of the inefficiency, see Supplementary Note A 3. This phenomenon of difference between cooperative and competitive decisions has been studied for a long time under different names. Economic inefficiency [37, 38] refers to a situation where total income, or social wealth, is not maximized. The problem of social cost [21, 22] is the divergence between private and social costs or value. Later developments include external effect [39], rent dissipation [40], market failure [41], and transaction cost [42]. Algorithmic game theory uses price of anarchy [18, 19] and price of stability for this inefficiency of equilibria. Despite the various terminology, the essence of the problem is the same: when individuals do not have incentive to maximize the total revenue, equilibrium naturally will differ from the optimum set, which by definition results in less total revenue. If people put a moderate weight towards the total outcome, much of the inefficiency can be avoided [5]. Here we propose the main takeaway for the case of multi-market oligopoly: if a property is heterogeneous in productivity, the owner cannot obtain the optimal rent by leasing to multiple tenants without contracting on their allocation of effort.

Now we point out how a driver would implement a search strategy. Picture a driver $i$ who is familiar with city traffic and hailer and driver distributions throughout a day. To earn more money, the driver has a plan on how much time to spend searching different places for hailers; the plan may vary for different time of day. At the beginning of $i$ 's shift, the driver heads to the region where the plan allocates the most search time. After delivering the first pickup, the driver is likely to be in a region with less planned search time. To avoid over-searching the current region, $i$ drives back to the preferred region. If $i$ goes through the preferred region without a pickup, the driver would circle around and continue the search, as long as the total search time within the region is not too long compared with the plan. The driver does not always search or immediately go back to the region with the highest planned search time, but would balance the allocation of realized search time to approximate the plan. But when $i$ drops off at a location with very little planned search time, the driver would directly head to a place nearby where the plan gives more search time, since a single pass would typically suffice for the drop-off location. Because the total search time is limited for any given shift, the driver would not be able to perfectly implement the strategy in one shift. But aggregated over time, the distribution of realized search time could reasonably approximate an intended strategy.

Driver's search strategy can be alternatively formalized as a Markov chain. That is, depending on the current location, the driver chooses probabilistically a neighboring location to search. If drivers are non-strategic, a null hypothesis for the Markov strategy would be random walk. However, fig. $1 \mathrm{~b}$ suggests that drivers tend to move back 

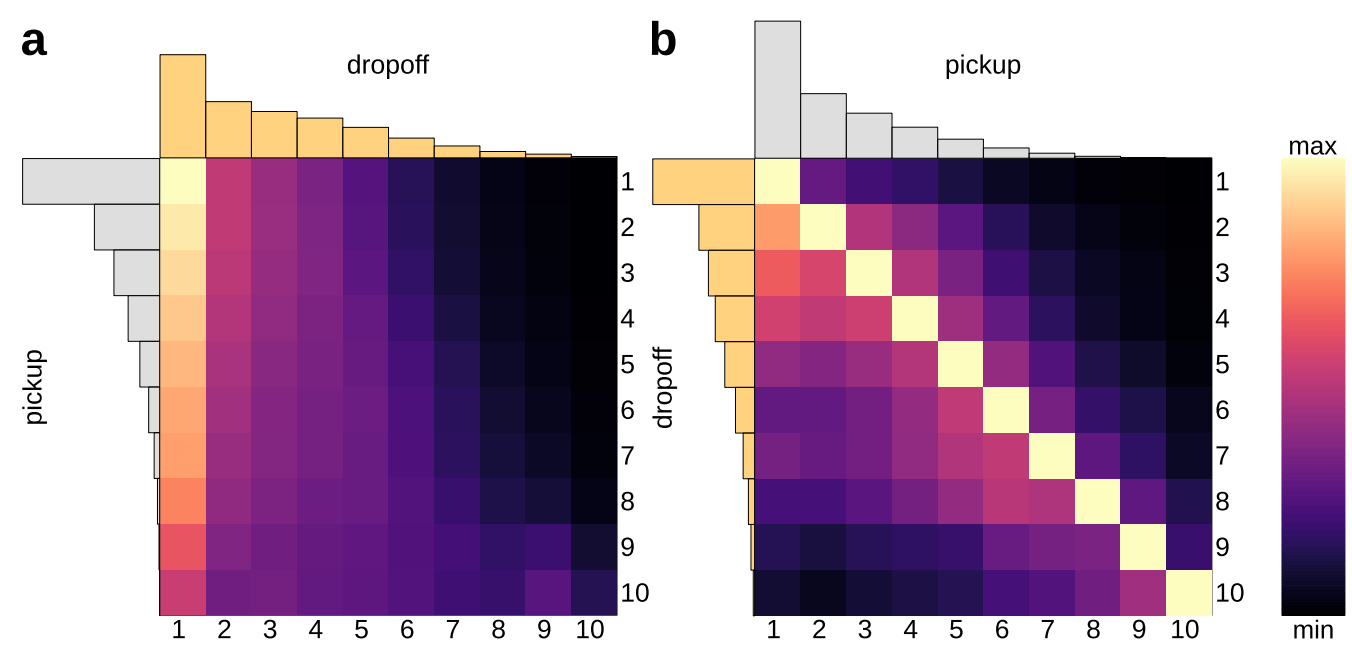

FIG. 4. Markov strategy. Using trip records in Spring 2011 and Spring 2012, Mon-Fri 6pm-7pm. a, trip origin-destination matrix among 10 equal-sized groups of street segments in decreasing order of pickups. Rows normalized by 1-norm to show transition probability. Margins show pickup and drop-off counts in each group. b, search start-end matrix among the groups. Rows normalized by max-norm.

to the area with more pickups. We test out this hypothesis in fig. 4. We see that, regardless of trip origin, locations with more pickups tend to be popular destinations as well. Overall the drop-off distribution is more spread out than the pickup distribution. On the other hand, the search matrix is diagonal dominated and skews towards more popular locations. In particular, group 1 accounts for $42 \%$ of pickups and $32 \%$ of drop-offs, and comparatively very few drivers find their next pickup in other groups. As the drop-off location gets less popular, the skew away from less popular groups and towards more popular ones become more prominent. Because search time is typically short in the PM peak, most drivers do not need to drive far to find passengers. Although a Markovian search strategy may be simple to describe and implement, it is difficult to estimate and does not allow a simple thermodynamic interpretation. Moreover, it is equivalent to our definition of search strategy. For details of the Markov strategy, see Supplementary Note A 4.

\section{METHODS}

\section{A. Taxi trip records}

The New York City (NYC) Taxi and Limousine Commission (TLC) started its Taxicab Passenger Enhancement Program (TPEP) in late 2008, which collects electronic trip record of its Medallion taxis (aka yellow cabs). TLC releases TPEP records to the public per the Freedom of Information Law of New York State. We have gathered the records from 2009 to 2013, the first five calendar years since TPEP devices were installed in all 13,237 Medallion taxis. The data set contains over 870 million trips and 50,297 frequent drivers. Each trip record contains medallion ID (for vehicles), hack license (for drivers), latitude, longitude and time stamp of pickup and drop-off, trip distance, fare amounts, and other attributes. We use the ID fields to link a taxi between consecutive trips, and derive new attributes for use in our study. The original and processed data are available for reuse at $[43]$.

\section{B. Road network and map matching}

We use OpenStreetMap (OSM) data for the public nonfreeway vehicular road network in NYC. Specifically, we include OSM ways whose highway tag take one of the following values: trunk, primary, secondary, tertiary, unclassified, residential. To make the road network strongly connected, we removed tunnels, bridges, and link roads. The filtered OSM map has 8,928 locations and 11,458 edges. We use Open Source Routing Machine (OSRM) to create a compressed graph of 6,001 edges. We exploit another module in OSRM to match GPS locations to the nearest segment, where longitudes and latitudes are transformed in Mercator projection for isotropic local scales of distance. The modified code is available at https://github.com/rudazhan/osrm-backend.

\section{Appendix A: Supplementary notes}

\section{Taxi driver decision making}

The transportation decision of a taxi driver can be simply expressed as: taxi drivers maximize their income by choosing their driving strategy. We ignore the exit decision of taxi drivers, and assume that individuals who drive a taxi can earn at least as much income as their cost, i.e. their alternative income. When this condition 
does not hold, rational individuals would not be driving a taxi. We show in the following that driver's objective function is strategically equivalent to trip revenue, and formalize driver's decision as an optimization problem.

Income structure of a taxi driver differs by the property rights of the taxi in use. Owner-drivers are Medallion owners who also drive their taxis, so they have no lease to pay. Drivers of driver-owned vehicle (DOV) lease a Medallion from fleets, agents, or Medallion owners, and either own or finance the purchase of the vehicle, at different lease costs. Other drivers lease both the Medallion and the vehicle. In any kind of such leases, the driver pays a fixed amount of money either per shift which lasts 12 hours, or per week in longer-term leases. (See TLC Rules §58-21: Leasing a Taxicab or Medallion.) The lease may optionally include gasoline surcharge, also a fixed amount, since 2012-09-30. (See TLC lease cap rules change. https://www1.nyc.gov/assets/tlc/ downloads/pdf/archived_public_notices/lease_ cap_rules_passed.pdf) Taxi lease type can be inferred from driver names on the taxi's rate card: if a taxi has named drivers, its owner typically uses long-term lease; if it has unspecified driver, its owner typically uses shift lease. Table I shows the number of NYC taxis in 2005 by their manager and driver types, derived from [44].

TABLE I. NYC taxis by manager-driver type, 2005

\begin{tabular}{|c|c|c|c|}
\hline & Owner-driver & Named driver & Unspecified \\
\hline Owner & 3730 & 1210 & - \\
\hline Fleet & - & 1481 & 635 \\
\hline Agent & - & 1435 & 4305 \\
\hline
\end{tabular}

Taxi drivers also pay for fuel usage, which depends on vehicle model, vehicle speed and acceleration, air temperature, and air conditioning. As of vehicle model, after the 2008-05-02 TLC auction, 275 of the 13237 Medallions are restricted to alternative fuel vehicles, but many unrestricted Medallion owners voluntarily converted to cleanfuel vehicles (see Table III, data from TLC 2008-2013 Annual Reports http://www.nyc.gov/html/tlc/html/ archive/annual.shtml). For gasoline/hybrid light passenger vehicles operating at urban traffic speed (16-40 $\mathrm{km} / \mathrm{h}$, or 10-25 mph), fuel consumption per hour is almost constant, see [45]. This means that fuel cost per service time can be seen as a constant for each taxicab regardless of speed - we do not consider taxis parked by the curb with engine off actively in service. Even without this observation, fuel cost per service time would still be approximately constant for a driver in one shift, as long as the driver has consistent driving speeds and acceleration patterns.

A taxi driver earns the remaining fare and tips after paying for lease, fuel, or both. Formally, the hourly income $u_{i}$ of driver $i$ derives from hourly trip revenue $\pi_{i}$, minus hourly fuel cost $f_{i}$, minus amortized hourly lease payment $r_{i}$ :

$$
u_{i}=\pi_{i}-f_{i}-r_{i}
$$

TABLE II. NYC taxis by vehicle fuel type

\begin{tabular}{|c|c|c|c|c|}
\hline End of year & Gasoline & ectric & esel & \\
\hline 2007 & 12422 & 728 & 0 & - \\
\hline 2008 & 11394 & 1843 & 0 & - \\
\hline 2009 & 10177 & 3043 & 17 & - \\
\hline 2010 & 9029 & 4185 & 19 & 4 \\
\hline 2011 & 7540 & 5681 & 14 & 2 \\
\hline 2012 & 6455 & 6769 & 10 & 3 \\
\hline 2013 & 5320 & 7905 & 9 & 3 \\
\hline
\end{tabular}

The amortized hourly lease payment by the driver is $r_{i}=R_{i} / T_{i}$, where $T_{i}$ denotes driver total service time during the lease term, and $R_{i}$ denotes lease payment, i.e. rent of the Medallion taxicab. Depending on the lease, $f_{i}$ or $r_{i}$ may be zero. Since $f_{i}$ and $r_{i}$ are constant for driver $i$ in any given shift, they do not affect the driver's driving strategy. Thus, driver's objective is strategically equivalent to trip revenue $\pi_{i}$. We note that although vehicle maintenance is another cost to drivers who own the vehicle, it is not relevant to the driver strategy of our interest.

To define taxi drivers' driving strategy, we first analyze taxi transportation. Taxis in service are either vacant or occupied: when vacant, drivers search the streets for hailers; when occupied, drivers take the passengers to their destination. Taxi drivers can freely choose how they spend their search time over the street network. Once they find hailers, drivers will stop searching and pick them up. (In real life, not all taxi drivers pick up every hailer they meet. They may discriminate hailers based on the destination, race, or other factors, due to profitability, security, or end-of-shift concerns. See NYC 311 records for complaints about taxis service denial.) Taxi fare rate is set by the city government, which may be metered or has a flat rate, depending on the destination. Under flat rate, drivers are best off taking the fastest path. Metered rates charge by distance or duration, based on a speed threshold, which are typically set such that drivers have no incentive to drive slow. Although drivers do have an incentive to take routes longer than the fastest path, passengers typically are motivated to supervise trip duration. In case of driver fraud, detouring is not a common strategy [46]. Thus, we assume that taxi driver's delivery strategy is to take passengers to their destination via the fastest path, so trip duration between two specific locations only depend on traffic speed. We can see that the only strategic element for taxi drivers is how they allocate their search time.

Now we formalize drivers' driving strategy. Let $N$ be the set of taxi drivers currently in service. Let $G=(V, E)$ be the road network within the urban area being studied, where $V$ is the set of intersections and dead ends, and $E$ is the set of street segments. Street segment $x \in E$ has length $l_{x}$, with traffic speed $v_{x}$ and taxi search speed $\tilde{v}_{x}$. Define demand rate $\mu_{d x y}$ as the frequency of hailers start hailing on segment $x$ who are going to segment $y$; such 
a group of hailers have impatience $\mu_{t x y}=1 / \mathbb{E} T_{x y}$, the reciprocal of hailer mean patience. Within a short time interval, environment condition $\mathcal{E}=\left(\mathbf{v}, \boldsymbol{\mu}_{d}, \boldsymbol{\mu}_{t}\right)$ can be considered as constant, where $\mathbf{v}$ is the vector of traffic speeds, and $\boldsymbol{\mu}_{d}$ and $\boldsymbol{\mu}_{t}$ are matrices of hailer demand and impatience. Strategy for driver $i$ can be defined as the spatial distribution of supply rates $\boldsymbol{\mu}_{s i}$, where $\mu_{s i x}=$ $\left(\boldsymbol{\mu}_{s i}\right)_{x}$ is the frequency at which driver $i$ enters segment $x$ as a vacant taxi. Equivalently, driver strategy can be defined as the distribution of driver's search time per unit time:

$$
\frac{t_{s i x}}{t}=\frac{l_{x}}{\tilde{v}_{x}} \mu_{s i x}
$$

This shows that on each segment, driver search time is linearly related to driver supply rate. Define pickup rate $\mu_{\text {pixy }}$ as the frequency at which driver $i$ picks up passengers on $x$ going to $y$. These attributes naturally aggregates on each segment: $\mu_{p x}=\sum_{i} \sum_{y} \mu_{p i x y}, \mu_{s x}=$ $\sum_{i} \mu_{s i x}, \mu_{d x}=\sum_{y} \mu_{d x y}$, and $\mu_{t x}=1 / \mathbb{E} T_{x}$. Pickup rate can thus be expressed as a function of supply rate, demand rate and hailer impatience: $\mu_{p x}\left(\mu_{s x}, \mu_{d x}, \mu_{t x}\right)$. [32] proposed a class of pickup models and proved that the pickup rate functions are increasing, strictly concave, and arbitrarily differentiable, with respect to supply rate; for three representative models, analytical forms of the pickup rate functions are also provided.

We now relate driver strategy with driver revenue. Let $\Pi_{x y}$ be the revenue of a single trip from $x$ to $y$, which only depends on traffic speeds $\mathbf{v}$. We can write hourly revenue originated on $x$ as $\pi_{x}=\sum_{y} \Pi_{x y} \mu_{p x y}$ and average revenue of a trip originated on $x$ as $\bar{\Pi}_{x}=\pi_{x} / \mu_{p x}$. Assume patience and destination are approximately uncorrelated for hailers with the same origin, which means $\forall x, y \in$ $E, \mu_{t x} \approx \mu_{t x y}$. Then hailers on the same segment have an equal chance of being picked up regardless of their destination:

$$
\forall x \in E, \mu_{p x y} \propto \mu_{d x y}, \forall y \in E
$$

Thus, the average revenue for a trip originated on $x$ only depends on traffic speeds and demand rates: $\bar{\Pi}_{x}\left(\mathbf{v}, \boldsymbol{\mu}_{d x}\right)=\sum_{y} \Pi_{x y} \mu_{d x y} / \mu_{d x}$. Since drivers are assumed not to discriminate hailers:

$$
\forall i \in N, \forall x \in E, \mu_{p i x y} \propto \mu_{p x y}, \forall y \in E
$$

Driver revenue originated on a segment $\pi_{i x}=\sum_{y} \Pi_{x y} \mu_{p i x y}$ can thus be written as $\pi_{i x}=\sum_{y} \Pi_{x y} \mu_{p x y} \mu_{p i x} / \mu_{p x}=\bar{\Pi}_{x} \mu_{p i x}$. Since each pass of a vacant taxi has an equal chance of picking up a hailer regardless of the driver:

$$
\forall x \in E, \mu_{p i x} \propto \mu_{\text {six }}, \forall i \in N
$$

We have $\pi_{i x}=\bar{\Pi}_{x} \mu_{p i x}=\bar{\Pi}_{x} \mu_{p x} \mu_{s i x} / \mu_{s x}$. Driver hourly trip revenue can thus be expressed with explicit function dependency as:

$$
\pi_{i}=\sum_{x \in E} \pi_{i x}=\sum_{x \in E} \bar{\Pi}_{x}\left(\mathbf{v}, \boldsymbol{\mu}_{d x}\right) \mu_{p x}\left(\mu_{s x}, \mu_{d x}, \mu_{t x}\right) \frac{\mu_{s i x}}{\mu_{s x}}
$$

A more analytically convenient definition of driver strategy is driver's allocation of service time. Service time $t_{i x}=t_{s i x}+t_{p i x}$ is the total time driver $i$ spends searching and delivering trips originated on $x$ during a period of time $t$. The rationale of using service time distribution as driver strategy instead of supply rate or search time is that: service time is a conserved quantity and identical for all drivers; meanwhile, service time is monotonic in supply rate and preserves properties of the pickup rate function. Let $t_{x y}$ be the trip duration from $x$ to $y$, which only depends on traffic speeds $\mathbf{v}$. The average duration of a trip originated on $x$ is $\bar{t}_{x}\left(\mathbf{v}, \boldsymbol{\mu}_{d x}\right)=\sum_{y} t_{x y} \mu_{d x y} / \mu_{d x}=$ $\sum_{y} t_{x y} \mu_{p x y} / \mu_{p x}$, with reasoning similar to average trip revenue $\bar{\Pi}_{x}$. The proportion of time driver $i$ spends delivering trips originated on $x$ is thus $t_{p i x} / t=\sum_{y} t_{x y} \mu_{p i x y}=$ $\sum_{y} t_{x y} \mu_{p x y} \mu_{p i x} / \mu_{p x}=\bar{t}_{x} \mu_{p i x}=\bar{t}_{x} \mu_{p x} \mu_{s i x} / \mu_{s x}$, with reasoning similar to $\pi_{i x}$. Together with Equation A2, the proportion of service time driver $i$ allocates on $x$ can thus be written as:

$$
s_{i x}=\frac{t_{s i x}+t_{p i x}}{t}=\left(\frac{l_{x}}{\tilde{v}_{x}}+\bar{t}_{x} \frac{\mu_{p x}}{\mu_{s x}}\right) \mu_{s i x}
$$

This shows that on each segment, driver service time is also linearly related to driver supply rate: $\forall x \in E, s_{i x} \propto$ $\mu_{s i x}, \forall i \in N$. From Equation A4, service time on a segment $s_{x}=\mu_{s x} l_{x} / \tilde{v}_{x}+\mu_{p x} \bar{t}_{x}$. With pickup rate function $\mu_{p x}\left(\mu_{s x}, \mu_{d x}, \mu_{t x}\right)$ and constant environment condition $\mathcal{E}$, pickup rate is implicitly a function of service time: $\mu_{p x}\left(s_{x}, \mathcal{E}\right)$. Each taxi driver must allocate all the service time among the street segments: $\sum_{x} t_{i x}=t$, or equivalently $\sum_{x} s_{i x}=1$. The driving strategy of taxi driver $i$ is thus $\mathbf{s}_{i} \in S_{i}$, where the strategy space $S_{i}=\Delta^{|E|-1}$, a simplex of dimension one less than the number of segments. Now we can formally write the optimization problem of a taxi driver:

$$
\begin{array}{cl}
\text { maximize } & \sum_{x \in E} \bar{\Pi}_{x}\left(\mathbf{v}, \boldsymbol{\mu}_{d x}\right) \mu_{p x}\left(s_{x}, \mathcal{E}\right) \frac{s_{i x}}{s_{x}} \\
\text { subject to } & \mathbf{s}_{i} \geq 0 \\
& \mathbf{s}_{i} \cdot \mathbf{1}=1
\end{array}
$$

Now we prove that pickup rate $\mu_{p x}\left(s_{x}, \mathcal{E}\right)$ is also increasing, strictly concave, and arbitrarily differentiable with respect to $s_{x}$. With constant environment condition $\mathcal{E}$, the implicit function can be abstracted to $z=a x+b y$, where $z=s_{x}, x=\mu_{s x}, y=\mu_{p x}, a=l_{x} / \tilde{v}_{x}$, and $b=\bar{t}_{x}$; $y(x)$ is increasing, strictly concave, and arbitrarily differentiable, while $a, b>0$ are constants. Our proposition is thus equivalent to: $y(z)$ is also increasing, strictly concave, and arbitrarily differentiable. Differentiability is simply preserved by the linear relation. Since $z(x)=a x+$ $b y(x)$ is increasing, its inverse $x(z)$ is thus also increasing; by composition, $y(z)=y(x(z))$ is also increasing. By implicit differentiation, $\mathrm{d} y / \mathrm{d} z=y^{\prime}(x) /\left(a+b y^{\prime}(x)\right)$, and thus $\mathrm{d}^{2} y / \mathrm{d} z^{2}=a y^{\prime \prime}(x) /\left(a+b y^{\prime}(x)\right)^{3}$. Since $y^{\prime}(x)>0$ and $y^{\prime \prime}(x)<0, y^{\prime \prime}(z)<0$, which means $y(z)$ is also strictly concave. 


\section{Multi-market oligopoly}

In this section we formalize the game of multi-market competition among firms of equal capacity, and prove that the game has Nash equilibrium (NE), which is symmetric and essentially unique in that marginal player payoffs are uniform across all invested markets.

We use subscript $x$ to denote a market, or product; subscript $i$ for a firm, or player; subscript $-i$ for opponents of firm $i$. Boldface denotes a vector; single subscript indicates summation. Conditions in parentheses are optional.

Game setup of multi-market oligopoly. For firms $i \in$ $N,|N|=n$, each distributing a unit of resources over markets $x \in E,|E|=m$ :

1. Total payoff in a market $u_{x}\left(s_{x}\right), s_{x} \geq 0, u_{x}(0)=$ 0 , is (increasing) non-decreasing, differentiable, and (strictly) concave;

2. Payoff per investment in a market $p_{x}\left(s_{x}\right)=u_{x} / s_{x}$, $s_{x}>0$, is (decreasing) non-increasing; not necessarily convex;

3. Player payoff in a market $u_{i x}\left(s_{i x} ; s_{-i x}\right)=p_{x}\left(s_{x}\right) s_{i x}$, $s_{i x} \in[0,1], s_{-i x}=\sum_{j \neq i} s_{j x} \in[0, n-1]$;

4. Player payoff $u_{i}\left(\mathbf{s}_{i} ; \mathbf{s}_{-i}\right)=\sum_{x} u_{i x}\left(s_{i x} ; s_{-i x}\right), \mathbf{s}_{i} \in$ $S_{i}=\Delta^{m-1}, \mathbf{s}_{-i}=\sum_{j \neq i} \mathbf{s}_{j} \in S_{-i}=(n-1) \Delta^{m-1} ;$ Here $\Delta^{m-1}=\left\{\mathbf{v} \in \mathbb{R}^{m} \mid \mathbf{v} \geq 0, \mathbf{v} \cdot \mathbf{1}=1\right\}$ is the $(m-1)$-dimensional simplex.

5. Marginal player payoff in a market at equilibrium $\phi_{x}\left(s_{x}\right)=p_{x}\left(s_{x}\right)+p_{x}^{\prime}\left(s_{x}\right) s_{x} / n$, or equivalently $\phi_{x}\left(s_{x}\right)=u_{x}^{\prime}\left(s_{x}\right) / n+(1-1 / n) u_{x}\left(s_{x}\right) / s_{x}$, is (positive) non-negative, (decreasing) non-increasing;

6. Potential function $\Phi(\mathbf{s})=\sum_{x} \int_{0}^{s_{x}} \phi_{x}(t) \mathrm{d} t$, $\mathbf{s} \in \quad n \Delta^{m-1}, \underset{\text { thus }}{s_{x}} \Phi(\mathbf{s})=$ $\sum_{x}\left[u_{x}\left(s_{x}\right) / n+(1-1 / n) \int_{0}^{s_{x}} u_{x}(t) / t \mathrm{~d} t\right] ;$

Multi-market oligopoly is similar to Cournot oligopoly [47], but differs in significant ways. In Cournot oligopoly, each player chooses a production level of the same product, whose marginal return decreases with total production; while the multi-market oligopoly can be seen as a multi-product Cournot game, where all players have the same total productivity. Formally, Cournot oligopoly can be written as: $G_{c}=\{N, Q, \mathbf{u}\}$, where player strategy $q_{i} \in Q_{i}=\mathbb{R}_{\geq 0}$, and player payoff function $u_{i}\left(q_{i}, q_{-i}\right)=$ $p(q) q_{i}-c q_{i}$; price $p(q)$ is a decreasing function on total productivity $q=\sum_{i} q_{i}$, and marginal cost $c$ is assumed to be constant. The multi-market oligopoly instead has $m$ products, and each player distributes one unit of resource $s$ among the products, earning payoff from all products invested.

To prove that multi-market firms of the same capacity have a unique and symmetric NE, we follow a list of propositions shown below. Before getting into the details, we point out the keys to the proof: convex game guarantees NE exists; equal capacity leads to symmetry; and monotonic marginal payoffs provide a unique solution.
Proposition 1. $\Phi(\mathbf{s})$ is (strictly) concave.

Proof. Let $P_{x}\left(s_{x}\right)=\int_{0}^{s_{x}} p_{x}(t) \mathrm{d} t$. Since $P_{x}\left(s_{x}\right)$ is a differentiable real function with a convex domain, it is (strictly) concave if and only if it is globally (strictly) dominated by its linear expansions: $\forall s_{0}>0, \forall s_{x} \geq$ $0, s_{x} \neq s_{0}$,

$$
\begin{aligned}
& P_{x}\left(s_{x}\right)-\left[P_{x}\left(s_{0}\right)+p_{x}\left(s_{0}\right)\left(s_{x}-s_{0}\right)\right] \\
= & \int_{s_{0}}^{s_{x}} p_{x}(t) \mathrm{d} t-p_{x}\left(s_{0}\right)\left(s_{x}-s_{0}\right) \\
= & \int_{s_{0}}^{s_{x}} p_{x}(t)-p_{x}\left(s_{0}\right) \mathrm{d} t \leq 0
\end{aligned}
$$

This is true because $p_{x}\left(s_{x}\right)$ is (decreasing) non-increasing. Because $\Phi(\mathbf{s})$ is a positive linear transformation of $P_{x}\left(s_{x}\right)$ and $u_{x}\left(s_{x}\right)$ which is also (strictly) concave, it implies that $\Phi(\mathbf{s})$ is (strictly) concave on the non-negative cone $\mathbb{R}_{\geq 0}^{m}$. Because simplex $n \Delta^{m-1}$ is a convex subset of the non-negative cone $\mathbb{R}_{\geq 0}^{m}$, it implies that $\Phi(\mathbf{s})$ is (strictly) concave on the simplex $n \Delta^{m-1}$. This proves Proposition 1.

Proposition 2. $u_{i}\left(\mathbf{s}_{i} ; \mathbf{s}_{-i}\right)$ is (strictly) concave, $\forall i, \forall \mathbf{s}_{-i} \in S_{-i}$.

Proof. Because simplex $S_{i}$ is a convex subset of the nonnegative cone $\mathbb{R}_{\geq 0}^{m}$, if $u_{i}\left(\mathbf{s}_{i} ; \mathbf{s}_{-i}\right)$ is (strictly) concave on $\mathbb{R}_{\geq 0}^{m}, \forall i, \forall \mathbf{s}_{-i} \in \bar{S}_{-i}$, then $u_{i}\left(\mathbf{s}_{i} ; \mathbf{s}_{-i}\right)$ is also (strictly) concave on $S_{i}, \forall i, \forall \mathbf{s}_{-i} \in S_{-i}$. It suffices to prove the former statement without constraints on opponent strategies: $u_{i}\left(\mathbf{s}_{i} ; \mathbf{s}_{-i}\right)$ is (strictly) concave on $\mathbb{R}_{\geq 0}^{m}, \forall i, \forall \mathbf{s}_{-i} \in \mathbb{R}_{\geq 0}^{m}$. Because $u_{i}\left(\mathbf{s}_{i} ; \mathbf{s}_{-i}\right)$ is a positive linear transformation of $u_{i x}\left(s_{i x} ; s_{-i x}\right), x \in E$, it suffices if $u_{i x}\left(s_{i x} ; s_{-i x}\right)$ is (strictly) concave on $\mathbb{R}_{\geq 0}, \forall x, \forall i, \forall s_{-i x} \geq 0$. To simplify notations, this is equivalent to $u_{i x}(s ; c)=p_{x}(s+c) s$ (strictly) concave on $\mathbb{R}_{\geq 0}, \forall x, \forall c \geq 0$. This can be proved by definition, and we do not include the proof here because it is straightforward but tedious. The key to this proof is that $u_{x}\left(s_{x}\right)$ is (strictly) concave and $p_{x}\left(s_{x}\right)$ is (decreasing) non-increasing; either of the optional conditions can guarantee strict concavity. This proves Proposition 2 .

Proposition 3. Multi-market oligopoly is a convex game.

Proof. A convex game is a game where each player has a convex strategy space and a concave payoff function $u_{i}\left(s_{i} ; s_{-i}\right)$ for all opponent strategies. In this game, player strategy space is the same simplex $S_{i}=\Delta^{m-1}$ for all players, which is convex. Together with Proposition 2, this is proves Proposition 3.

Proposition 4. Multi-market oligopoly has NE, (all strict).

Proof. A convex game has NE if it has a compact strategy space and continuous payoff functions, see [48]. Because the product space of simplices is compact, this game has 
a compact strategy space $S \equiv \prod_{i} S_{i}=\prod_{i} \Delta^{m-1}$. Because $u_{x}\left(s_{x}\right)$ is continuous $\forall x$, player payoff $u_{i}(\mathbf{s})$ is thus continuous $\forall i$. This game thus has NE. If $u_{i}\left(\mathbf{s}_{i} ; \mathbf{s}_{-i}\right)$ is strictly concave, all NEs are strict. This proves Proposition 4 .

Proposition 5. Multi-market oligopoly can only have symmetric NE.

Proof. Given a NE $\mathbf{s}^{*}$, for all player $i$, equilibrium strategy $\mathbf{s}_{i}^{*}$ solves the convex optimization problem:

$$
\begin{array}{ll}
\operatorname{maximize} & u_{i}\left(\mathbf{s}_{i} ; \mathbf{s}_{-i}^{*}\right) \\
\text { subject to } & \mathbf{s}_{i} \geq 0 \\
& \mathbf{s}_{i} \cdot \mathbf{1}=1
\end{array}
$$

Since this convex optimization problem is strictly feasible, by Slater's theorem, it has strong duality. Since the objective function $u_{i}\left(\mathbf{s}_{i} ; \mathbf{s}_{-i}^{*}\right)$ is differentiable, the KarushKuhn-Tucker (KKT) theorem states that optimal points of the optimization problem is the same with the solutions of the KKT conditions:

$$
\begin{array}{ll}
\nabla u_{i}+\boldsymbol{\lambda}_{i}-\nu_{i} \mathbf{1}=0 & \text { (saddle point) } \\
\mathbf{s}_{i} \geq 0 & \text { (primal constraint 1) } \\
\boldsymbol{\lambda}_{i} \geq 0 & \text { (dual constraint) } \\
\mathbf{s}_{i} \circ \boldsymbol{\lambda}_{i}=0 & \text { (complementary slackness) } \\
\mathbf{s}_{i} \cdot \mathbf{1}=1 & \text { (primal constraint 2) }
\end{array}
$$

Here operator $\circ$ denotes the Hadamard product: $(x \circ$ $y)_{i}=x_{i} y_{i}$. Given the saddle point conditions $\partial u_{i} / \partial s_{i x}+$ $\lambda_{i x}-\nu_{i}=0, \forall x$, the dual constraint implies that the marginal payoff for player $i$ in market $x$ is bounded above: $\partial u_{i} / \partial s_{i x} \leq \nu_{i}, \forall x$. If player $i$ invests in market $x, s_{i x}>0$, by complementary slackness the upper bound is tight, which means the marginal payoffs for player $i$ are uniform in all markets $i$ invests. Together, marginal player payoffs at equilibrium have relation:

$$
\frac{\partial u_{i}}{\partial s_{i y}} \leq \frac{\partial u_{i}}{\partial s_{i x}}=\nu_{i}, \quad \forall i, \forall x, y, s_{i x}^{*}>s_{i y}^{*} \geq 0
$$

Since $\partial u_{i} / \partial s_{i x}=p_{x}\left(s_{x}\right)+p_{x}^{\prime}\left(s_{x}\right) s_{i x}$, and $p_{x}^{\prime}<0$, this is equivalent to $p_{x}\left(s_{x}^{*}\right) \leq \nu_{i}+\left|p_{x}^{\prime}\left(s_{x}^{*}\right)\right| s_{i x}^{*}, \forall x$, with equality if $s_{i x}^{*}>0$. If player $i$ invests more in market $x$ than player $j$ does, $s_{i x}^{*}>s_{j x}^{*} \geq 0$, this implies $\nu_{i}+\left|p_{x}^{\prime}\left(s_{x}^{*}\right)\right| s_{i x}^{*} \leq$ $\nu_{j}+\left|p_{x}^{\prime}\left(s_{x}^{*}\right)\right| s_{j x}^{*}$. But because the players have the same capacity, player $i$ must have invested less in some market $y$ than player $j$ does: $s_{j y}^{*}>s_{i y}^{*} \geq 0$, which implies $\nu_{j}+\left|p_{y}^{\prime}\left(s_{y}^{*}\right)\right| s_{j y}^{*} \leq \nu_{i}+\left|p_{y}^{\prime}\left(s_{y}^{*}\right)\right| s_{i y}^{*}$. Together, these inequalities imply $\left|p_{x}^{\prime}\left(s_{x}^{*}\right)\right|\left(s_{i x}^{*}-s_{j x}^{*}\right)+\left|p_{y}^{\prime}\left(s_{y}^{*}\right)\right|\left(s_{j y}^{*}-s_{i y}^{*}\right) \leq 0$. This contradicts our assumption on player resource allocation, thus all players must have the same strategy in equilibrium. This proves Proposition 5.

Proposition 6. Multi-market oligopoly have a (unique) essentially unique NE, in that marginal player payoffs are uniform across all invested markets.
Proof. From Proposition 4 and 5, multi-market firms have NE, which are symmetric. For a symmetric NE $\mathbf{s}^{*}$, player strategy $\mathbf{s}_{i}^{*}=\mathbf{s}^{*} / n$, and marginal player payoffs in invested markets are the same for all players: $\nu_{i}=\nu, \forall i$. Now the relation among equilibrium marginal player payoffs can be rewritten as: $p_{x}\left(s_{x}^{*}\right)+p_{x}^{\prime}\left(s_{x}^{*}\right) s_{x}^{*} / n \leq \nu, \forall x$, with equality in invested markets, $s_{x}^{*}>0$. Because $\phi_{x}\left(s_{x}\right)=p_{x}\left(s_{x}\right)+p_{x}^{\prime}\left(s_{x}\right) s_{x} / n$, this is equivalent to

$$
\phi_{x}\left(s_{x}^{*}\right) \leq \nu, \quad \forall x
$$

with equality in invested markets. Since $u_{x}\left(s_{x}\right)$ is a univariate differentiable (strictly) concave function, $u_{x}^{\prime}\left(s_{x}\right)$ is (decreasing) non-increasing. Because $p_{x}\left(s_{x}\right)$ is also (decreasing) non-increasing, $\phi_{x}\left(s_{x}\right)=u_{x}^{\prime}\left(s_{x}\right) / n+(1-$ $1 / n) p_{x}\left(s_{x}\right)$ is (decreasing) non-increasing. Define inverse function $\phi_{x}^{-1}: \mathbb{R}_{\geq 0} \rightarrow \mathbb{R}_{\geq 0}$, so that $\phi_{x}^{-1}(\nu)=0$ for $\nu>\phi_{x}^{-1}(0)$. The function is non-increasing (decreasing for $\left.\nu \leq \phi_{x}^{-1}(0)\right)$ and the equilibrium satisfies:

$$
s_{x}^{*}=\phi_{x}^{-1}(\nu), \quad \forall x
$$

Since total investment equals the number of players, $\sum_{x} s_{x}=n$, marginal player payoff in invested markets $\nu$ is determined by:

$$
\sum_{x} \phi_{x}^{-1}(\nu)=n
$$

Because the left-hand side of Equation A11 is decreasing for $\nu \leq \max _{x} \phi_{x}^{-1}(0)$ where the left-hand side is positive, the equation gives a unique solution $\nu$. Thus Equation A10 gives a (unique) essentially unique $\mathbf{s}^{*}$. Figure $1 \mathrm{~d}$ shows this process graphically. This proves Proposition 6 .

Proposition 7. The equilibrium of multi-market oligopoly is globally asymptotically stable under gradient adjustment process.

Proof. The gradient adjustment process [33] is a heuristic learning rule where players adjust their strategies according to the local gradient of their payoff functions, projected onto the tangent cone of player strategy space. Formally, gradient adjustment process is a dynamical system:

$$
\frac{\mathrm{d} \mathbf{s}_{i}}{\mathrm{~d} t}=P_{T\left(s_{i}\right)} \nabla_{i} u_{i}(\mathbf{s}), \quad \forall i
$$

Here $\nabla_{i}$ denotes the gradient with respect to player strategy $s_{i}, T\left(s_{i}\right)$ is the tangent cone of player strategy space $S_{i}$ at point $s_{i}$, and $P$ is the projection operator. For all interior points of the player strategy space, $P_{T\left(s_{i}\right)}$ is simply the centering matrix, $M_{1}=I-\mathbf{1 1}^{\mathrm{T}} / \mathrm{m}$. To prove that the dynamical system is globally asymptotically stable, we show that $V(\mathbf{s})=\Phi\left(\mathbf{s}^{*}\right)-\Phi(\mathbf{s})$ is a global Lyapunov function: a function that is positive-definite, continuously differentiable, and has negative-definite time derivative. From Proposition 1 and KKT theorem, the 
maximal points of the potential function $\Phi(\mathbf{s})$ is determined the same way as Equation A9. That is, the maximal set of the potential function is identical to the NE of multi-market oligopoly. This means $V(\mathbf{s})>0$ at nonequilibrium points, so $V(\mathbf{s})$ is positive-definite. $V(\mathbf{s})$ is clearly continuously differentiable, and its time derivative

$$
\begin{aligned}
\frac{\mathrm{d} V(\mathbf{s})}{\mathrm{d} t} & =-\nabla \Phi(\mathbf{s}) \cdot \frac{\mathrm{d} \mathbf{s}}{\mathrm{d} t} \\
& =-\sum_{x=1}^{m} \sum_{i=1}^{n}\left(\frac{\partial u_{i x}}{\partial s_{i x}}-\frac{1}{m} \sum_{y=1}^{m} \frac{\partial u_{i y}}{\partial s_{i y}}\right) \phi_{x} \\
& =-n \sum_{x=1}^{m}\left(\phi_{x}-\frac{1}{m} \sum_{y=1}^{m} \phi_{y}\right) \phi_{x} \\
& =-m n\left(\overline{\phi^{2}}-\bar{\phi}^{2}\right)
\end{aligned}
$$

Here $\bar{\phi}=\sum_{x=1}^{m} \phi_{x} / m$ and $\overline{\phi^{2}}=\sum_{x=1}^{m} \phi_{x}^{2} / m$. Thus, $\overline{\phi^{2}}-\bar{\phi}^{2} \geq 0$, with equality if and only if $\phi_{x}$ are all equal. From Equation A9, we can see that $\mathrm{d} V / \mathrm{d} t \leq 0$, with equality only at equilibrium points $\mathbf{s}^{*}$. We have thus shown that $V(\mathbf{s})$ is a global Lyapunov function of the dynamical system, which immediately implies Proposition 7 .

The stability result in Proposition 7 is only intended to show that under a simple and plausible learning rule, global asymptotic stability of Nash equilibrium is possible in multi-market oligopoly so that the equilibrium can be empirically observed. The gradient adjustment process adopted in this paper is not meant to be the exact learning rule used in real life, which is hard to determine. But compared with Bayesian or best-response learning rules, it is less demanding on the players as it does not require complete information of the game or long-term memory of the players. And even if some players adopt alternative, non-economic learning rules, the stability of the equilibrium may well be preserved. For example, new drivers may simply choose imitative learning [34, 35], or in other words "follow the older drivers". In this case the Nash equilibrium is still the stable focus as all players adopt the same strategy and the rational payoff-improving players adjust to the equilibrium. By imitative learning, new drivers save the possibly long process of strategy adjustment and quickly converge to the equilibrium strategy. This allows the equilibrium remain stable under an evolving set of drivers.

We note that the payoff in multi-market oligopoly is not strictly diagonally concave, so the uniqueness and stability results cannot follow [49]. But the eigenvalues of the Jacobian $\nabla(\mathrm{d} \mathbf{s} / \mathrm{d} t)$ are always negative, so local asymptotic stability at the equilibrium is guaranteed under gradient dynamics with individual-specific adjustment speeds. We also note that unlike the Cournot game, multi-market oligopoly is not an aggregate game defined in [50] or later generalizations, because player strategies are multi-dimensional. Thus it does not inherit the stability under discrete-time best-response dynamics. Multimarket oligopoly is also not a potential game and thus does not inherit the general dynamic stability properties in [51]. Instead, we provided a "potential function" that is a global Lyapunov function for the gradient dynamics.

\section{Inefficiency of the equilibrium}

We notice that in multi-market oligopoly the total payoff $u(\mathbf{s})=\sum_{i \in N} u_{i} \neq \Phi(\mathbf{s})$, which means total payoff is generally not maximized in Nash equilibrium, thus not socially optimal. In fact, if total payoff is maximized, then $\partial u_{x} / \partial s_{x} \geq \partial u_{y} / \partial s_{y}, \forall x, y \in E, s_{x}>0$, which means marginal payoff are the same for all invested markets. Compare with Equation A9 and the definition of $\phi_{x}\left(s_{x}\right)$, a weighted average of marginal and average payoff is balanced instead. With $n \gg 1$ in the case of NYC taxi system, we have $\phi \approx u_{x} / s_{x}$. So at equilibrium the average segment revenue per service time are effectively the same for all searched segments.

This is similar to the Cournot game. The total payoff in the Cournot game is $u(q)=\sum_{i \in N} u_{i}=p(q) q-c q$. Assuming $p(q)$ differentiable, the social optimum is $u^{*}=$ $\left(p\left(q^{*}\right)-c\right) q^{*}$, where $q^{*}$ satisfies $p^{\prime}\left(q^{*}\right) q^{*}+p\left(q^{*}\right)=c$. The Nash equilibrium is $q_{i}=q^{\dagger} / n, \forall i \in N$, where $q^{\dagger}$ satisfies $p^{\prime}\left(q^{\dagger}\right) q^{\dagger} / n+p\left(q^{\dagger}\right)=c$. This makes $q^{\dagger}>q^{*}$ and $u\left(q^{\dagger}\right)<$ $u\left(q^{*}\right)$, so the Nash equilibrium is not social optimal, and decreases further as the number of player increases.

However, the Nash equilibrium of multi-market oligopoly is socially optimal if market payoffs are power functions of the same order: $u_{x}\left(s_{x}\right)=a_{x} s_{x}^{p}, a_{x}>0, p \in$ $(0,1)$. In this case the total payoff $u=\sum_{x \in E} a_{x} s_{x}^{p}$ and player payoff $u_{i}=\sum_{x \in E} a_{x} s_{x}^{p-1} s_{i x}$. At social equilibrium, $\partial u_{x} / \partial s_{x}=a_{x} p s_{x}^{p-1}$ is a constant for all markets $x \in E$. Since $\sum_{x \in E} s_{x}=\sum_{i \in N} \sum_{x \in E} s_{i x}=n$, social optimal strategy is $s_{x}^{*}=n a_{x}^{1 /(1-p)} / \sum_{y \in E} a_{y}^{1 /(1-p)}, \forall x \in E$. At Nash equilibrium, for all players $i \in N$, let $u_{i x}=$ $u_{x} s_{i x} / s_{x}$, then $\partial u_{i x} / \partial s_{i x}=a_{x}\left((p-1) s_{x}^{p-2} s_{i x}+s_{x}^{p-1}\right)$ is a constant for all markets $x \in E$. This means $\sum_{i \in E} \partial u_{i x} / \partial s_{i x}=(p-1+n) a_{x} s_{x}^{p-1}$ is a constant for all markets $x \in E$, which gives the same aggregate strategy $s_{x}^{\dagger}=n a_{x}^{1 /(1-p)} / \sum_{y \in E} a_{y}^{1 /(1-p)}, \forall x \in E$, so the Nash equilibrium is social optimal. Use the condition again, we find Nash equilibrium $s_{i x}^{\dagger}=s_{x}^{\dagger} / n, \forall i \in N, x \in E$.

\section{Markov strategy}

Here we show the equivalence of Markov strategy and search time allocation vector. A Markov strategy can be represented by a search transition matrix $Q_{x y}$, a right stochastic matrix that gives the transition probabilities from every segment $x$ to every neighboring segment $y$ while the driver is searching for passengers. Let $P_{x y}$ be the empirical pickup transition matrix, i.e. row- 
normalized trip origin-destination matrix. Let $p_{x}$ be the probability of pickup per search on a segment $x$, and $P_{x}=\operatorname{diag}\left\{p_{x}\right\}$. We note that $P_{x y}$ can be easily computed, see e.g. fig. 4a, while $p_{x}$ and $Q_{x y}$ can be computed if high resolution trajectory data is available. The equilibrium search time allocation vector $\mathbf{s}^{*}$ and the Markov strategy $Q_{x y}$ satisfy the equation:

$$
\mathbf{s}^{*}=\mathbf{s}^{*}\left[P_{x} P_{x y}+\left(I-P_{x}\right) Q_{x y}\right]
$$

To see this, think of an ensemble of vacant vehicles, each searching for pickup. After one time step, most continue to search on neighboring streets; some become occupied and exit the ensemble, and join the ensemble again after drop-off. Note that the drivers are non-interacting. Since we are only counting the allocation of search time, in longtime limit each driver would have the same allocation of search time, which equals the ensemble average at any time, and satisfies the equation as written. Because $p_{x}$ and $P_{x y}$ are determined by the environment condition, $Q_{x y}$ uniquely determines $\mathbf{s}^{*}$.
[1] A. Pentland, Social physics: how good ideas spread - the lessons from a new science (Scribe Publications, 2014).

[2] W. Pan, G. Ghoshal, C. Krumme, M. Cebrian, and A. Pentland, Urban characteristics attributable to density-driven tie formation, Nature Communications 4, 1961 (2013).

[3] Y. Yuan, A. Alabdulkareem, and A. S. Pentland, An interpretable approach for social network formation among heterogeneous agents, Nature Communications 9, 4704 (2018).

[4] M. C. Gonzalez, C. A. Hidalgo, and A.-L. Barabasi. Understanding individual human mobility patterns, Nature 453, 779 (2008).

[5] S. Colak, A. Lima, and M. C. Gonzalez, Understanding congested travel in urban areas, Nature Communications 7, 10.1038/ncomms10793 (2016).

[6] L. E. Olmos, S. Colak, S. Shafiei, M. Saberi, and M. C. Gonzalez, Macroscopic dynamics and the collapse of urban traffic, Proceedings of the National Academy of Sciences 115, 12654 (2018).

[7] W. Weidlich, Sociodynamics: a systematic approach to mathematical modelling in the social sciences, 1st ed. (Harwood Academic, Amsterdam, 2000).

[8] W. Weidlich and G. Haag, An integrated model of transport and urban evolution: with an application to a metropole of an emerging nation, 1st ed. (Springer-Verlag Berlin Heidelberg, 1999).

[9] D. Helbing, Quantitative sociodunamics: stochastic methods and models of social interaction processes, 2nd ed. (Springer, 2010).

[10] C. Castellano, S. Fortunato, and V. Loreto, Statistical physics of social dynamics, Reviews of Modern Physics 81, 591 (2009).

[11] L. M. A. Bettencourt, The origins of scaling in cities, Science 340, 1438 (2013).

[12] M. Lee, H. Barbosa, H. Youn, P. Holme, and G. Ghoshal, Morphology of travel routes and the organization of cities, Nature Communications 8, 2229 (2017).

[13] H. Barbosa, M. Barthelemy, G. Ghoshal, C. R. James, M. Lenormand, T. Louail, R. Menezes, J. J. Ramasco, F. Simini, and M. Tomasini, Human mobility: models and applications, Physics Reports 734, 1 (2018).

[14] M. Barthelemv, The statistical physics of cities, Nature Reviews Physics 1, 406 (2019).

[15] M. Mazzoli, A. Molas, A. Bassolas, M. Lenormand, P. Colet, and J. J. Ramasco. Field theory for recurrent mobility, Nature Communications 10, 3895 (2019).
[16] J. G. Wardrop, Some theoretical aspects of road traffic research, Proceedings of the Institution of Civil Engineers 1, 325 (1952).

[17] J. W. Forrester, Urban dynamics (M.I.T. Press, Cambridge, Mass., 1969).

[18] E. Koutsoupias and C. Papadimitriou, Worst-case equilibria, in STACS 99 (Springer Berlin Heidelberg, 1999) pp. 404-413.

[19] T. Roughgarden, Selfish routing, Ph.D. thesis, Department of Computer Science, Cornell University, Ithaca, New York (2002).

[20] D. Zhang, L. Sun, B. Li, C. Chen, G. Pan, S. Li, and $\mathrm{Z}$. Wu, Understanding taxi service strategies from taxi GPS traces. IEEE Transactions on Intelligent Transportation Systems 16, 123 (2015).

[21] A. C. Pigou, The economics of welfare, 1st ed. (Macmillan and Co., London, 1920).

[22] F. H. Knight. Some fallacies in the interpretation of social cost, Quarterly Journal of Economics 38, 582 (1924).

[23] M. J. Beckmann, C. B. McGuire, and C. B. Winsten, Studies in the economics of transportation (Yale University Press, 1956).

[24] D. E. Boyce, H. S. Mahmassani, and A. Nagurney, A retrospective on Beckmann, McGuire and Winsten's Studies in the Economics of Transportation, Papers in Regional Science 84, 85 (2005).

[25] E. L. Glaeser, Cities, agglomeration, and spatial equilibrium (Oxford University Prress, 2008).

[26] C. Cook, R. Diamond, J. Hall. J. A. List, and P. Over. The aender earninas aap in the aia economy: evidence from over a million rideshare drivers, Working Paper 24732 (National Bureau of Economic Research, 2018).

[27] Y. Zhang, B. Li, and K. Ramayya, Learning individual behavior using sensor data: the case of GPS traces and taxi drivers (2020).

[28] R. L. Meier, The metropolis as a transaction-maximizing system, Daedalus 97, 1292 (1968).

[29] A. Wilson, Entropy in urban and regional modelling: retrospect and prospect, Geographical Analysis 42, 364 (2010).

[30] E. Barbour, C. C. Davila, S. Gupta, C. Reinhart, J. Kaur, and M. C. Gonzalez, Planning for sustainable cities by estimating building occupancy with mobile phones, Nature Communications 10, 3736 (2019).

[31] R. Di Clemente, M. Luengo-Oroz, M. Travizano, S. Xu, B. Vaitla, and M. C. Gonzalez, Sequences of purchases in credit card data reveal lifestyles in urban populations, Nature Communications 9, 3330 (2018). 
[32] R. Zhang and R. Ghanem. Demand, supply, and performance of street-hail taxi. IEEE Transactions on Intelligent Transportation Systems , 1 (2019).

[33] K. J. Arrow and L. Hurwicz, Stability of the gradient process in n-person games, Journal of the Society for Industrial and Applied Mathematics 8, 280 (1960).

[34] A. E. Roth and I. Erev, Learning in extensive-form games: experimental data and simple dynamic models in the intermediate term, Games and Economic Behavior 8, 164 (1995).

[35] D. Fudenberg and D. K. Levine, Learning and equilibrium, Annual Review of Economics 1, 385 (2009).

[36] P. J. Smith, D. S. Rae, R. W. Manderscheid, and S. Silbergeld, Approximating the moments and distribution of the likelihood ratio statistic for multinomial goodness of fit, Journal of the American Statistical Association 76, 737 (1981).

[37] J. S. Mill, On liberty (J. W. Parker and Son, 1859).

[38] H. Sidgwick, Principles of political economy (Macmillan and co, 1883).

[39] J. E. Meade, External economies and diseconomies in a competitive situation, Economic Journal 62, 54 (1952).

[40] H. S. Gordon, The economic theory of a commonproperty resource: the fishery, Journal of Political Economy 62, 124 (1954).

[41] F. M. Bator, The anatomy of market failure, Quarterly Journal of Economics 72, 351 (1958).

[42] R. H. Coase, The problem of social cost, Journal of Law and Economics 3. 1 (1960).

[43] R. Zhang, New York City taxi trip records, 2009-2013 (2019).
[44] B. Schaller, The New York City taxicab fact book, Tech. Rep. (Schaller Consulting, 2006).

[45] S. C. Davis, S. W. Diegel, and R. G. Boundy, Transportation energy data book (Oak Ridge National Laboratory, 2017).

[46] L. Balafoutas, R. Kerschbamer, and M. Sutter, Seconddegree moral hazard in a real-world credence goods market, Economic Journal 127, 1 (2015).

[47] A. A. Cournot. Recherches sur les principes mathematiques de la theorie des richesses (chez L. Hachette, 1838).

[48] H. Nikaido and K. Isoda, Note on non-cooperative convex games, Pacific Journal of Mathematics 5, 807 (1955).

[49] J. B. Rosen, Existence and uniqueness of equilibrium points for concave n-person games, Econometrica 33, 520 (1965).

[50] R. Selten, Preispolitik der Mehrproduktenunternehmung in der statischen Theorie, Okonometrie und Unternehmensforschung No. v. 16 (Springer-Verlag, 1970).

[51] D. Monderer and L. S. Shaplev, Potential games, Games and Economic Behavior 14, 124 (1996).

\section{ACKNOWLEDGMENTS}

The authors thank Henry S. Farber of Princeton University and Abhishek Nagaraj of UC Berkeley for sharing NYC taxi trip records, and thank Ketan Savla, Sami F. Masri, Juan Carrillo, Matthew Kahn, Hashem Pesaran, and Geert Ridder of USC for helpful comments. Research is funded by National Science Foundation (NSF) under Grant No. 14-524 Resilient Interdependent Infrastructure Processes and Systems and, in part, by NSF Grant DMS1638521. 